\title{
The incidence of keratoconus and its association with ocular disorders in Lahore population
}

\author{
Shagufta Naz*, Nazia Ibrahim, Saima Sharif, Afia Iqbal, Nadia Sarwar, \\ Sumera Sajjad, and Afshan Kaleem \\ Department of Zoology, Lahore College for Women University, Lahore-Pakistan \\ *Corresponding author's email: shagufta6@gmail.com \\ Citation \\ Shagufta Naz, Nazia Ibrahim, Saima Sharif, Afia Iqbal, Nadia Sarwar, Sumera Sajjad, and Afshan Kaleem. The \\ incidence of keratoconus and its association with ocular disorders in Lahore population. Pure and Applied Biology. \\ Vol. 8, Issue 1, pp619-624. http://dx.doi.org/10.19045/bspab.2019.80001
}

Received: $20 / 08 / 2018$

Revised: $28 / 12 / 2018$

Accepted: 30/12/2018

Online First: 04/01/2019

\section{Abstract}

This study was conducted to check predominance of keratoconus in male and female of the various age groups in Pakistan and to check the severity of disease in keratoconus patients. Keratoconus is a progressive eye disease in which the normally round cornea thins and begins to bulge into a conelike shape. A cross-sectional, analytical study was performed between September 2016 to May 2017 on Keratoconus patients visiting Layton Rahmatullah Benevolent Trust Hospital and Sir Ganga Ram Hospital during their ophthalmic consultation. The techniques used for the diagnosis of Keratoconus were Visual Acuity by Snellen chart, Ophthalmoscopy, Orbscan corneal topography, and Slit lamp bio microscopy. Total 110 patients of Keratoconus were studied in which 62 were females and 48 were males. Patients developed both unilateral as well as bilateral conditions. Visual acuity from normal to high vision loss was also observed in Keratoconus patients. Different disorders with different percentage such as Munson's sign were present in 68 patients, Rizutti's sign was present in 30 patients, Vogt's striae were present in 26 patients and Fleischer ring was present in 23 patients. 19 patients had Atopic syndrome and 6 patients had Leber's Congenital Amaurosis were also associated with keratoconus.

Keywords: Cross-sectional; Keratoconus; Ophthalmoscopy; Orbscan corneal topography; Snellen chart; Slit lamp biomicroscopy

\section{Introduction}

Keratoconus is a chronic, progressive, noninflammatory disease of the cornea. It is a common primary ectasia of the cornea characterized by thinning and distortion of the cornea which results in the protrusion of the cornea and it assumes a conical shape. It usually affects all layers of the cornea. It develops in puberty and progresses until the third or fourth decade of life. So in the progressive stage, visual acuity lost which cannot be correct with spectacles. Keratoconus affects both genders and all ethnicities [1, 2]. The early forms of keratoconus go unnoticed unless corneal topography is performed but the severity of the disorder at the time when the progression stops can range from very mild irregular astigmatism to severe thinning, protrusion, and scarring which cause severe visual 
impairment that requires keratoplasty [3]. The most prominent early features of keratoconus are the thining of the corneal stroma, breaks in the Bowman layer and the formation of a Fleischer ring that is, an iron deposit in the basal epithelial cells. Vogt's striae; folds or fine vertical striations in the Descemet's membrane due to compression, Munson sign; in downward position $\mathrm{V}$-shape malformation of the lower eyelid due to corneal protrusion, Rizutti's sign; a bright conical reflection on nasal cornea when light is directed from the temporal side, acute hydrops; breaks in Descemet's membrane usually observes in advance stages of keratoconus [4]. Keratoconus associated with a number of ocular and systemic disorders; ocular associations include Leber's congenital amaurosis, Down syndrome, Ehlers-Danlos syndrome connective tissue disorder. The main risk factors are Atopic syndrome, eye rubbing, and rigid contact lens wear as well as a family history of keratoconus [5]. The cause of keratoconus is still unclear; it is clearly multifactorial involving both genetic and environmental factors. The disease may be triggered by environmental factors in genetically susceptible individuals. A strong indication of genetic influence comes from a positive family history of keratoconus in patients afflicted by the disease [6]. In a study performed in the Midlands area of the United Kingdom, Asians have Keratoconus prevalence of $4: 1$, while Caucasians showed 4.4:1 incidence of this disease. In other Study undertaken in Yorkshire, also in the United Kingdom, the incidence found was 7.5 times higher in Asians compared to Caucasians. The incidence rate of Keratoconus in Asian population is due to consanguineous relations, especially first-cousin marriages, which commonly take place in the Asian population of the area assessed [7].

\section{Material and methods}

A cross-sectional, analytical study was performed between September 2016 and May 2017 on all Keratoconus patients. Information about the patient's disease condition, progression, history, and clinical examination were collected by visiting Layton Rahmatullah Benevolent Trust Hospital, (LRBT) and Sir Ganga Ram hospital to obtain the data of patients affecting from Keratoconus. Data was also collected by filling the specially designed proforma which includes proper history, including a change in eyeglass prescription, patient age, gender, history of eye rubbing, medical problems, allergies, and sleep patterns.

Variables that were selected for further analysis and assessment are Age, Gender, Unilateral or Bilateral keratoconus, Munson sign, K-readings, Rizutti's sign, Vogt's striae, Fleischer ring, Syndrome (if any). For clinical evaluation of the patients of Keratoconus following methods are adopted Visual acuity, Direct and indirect Ophthalmoscopy, Corneal topography, Slit lamp biomicroscopy.

Chi-Square Test was performed and a comparison between different age groups and the number of patients of keratoconus was done, the significant level was 0.000 . Unilateral keratoconus, bilateral keratoconus, visual disability, history of eye rubbing and Munson's sign showed a significant level of 0.000. Rizzuti's sign was observed at a significant level of 0.002 , Vogt's striae were observed at a significant level of 0.003 . The significant level was $\mathrm{P} \leq 0.05$. Fleischer ring showed a significant level of 0.529 and different k-readings of patients of keratoconus showed a significant level of 0.095 . 


\section{Results and discussion}

In this study 110 patients of Keratoconus were recognized which included 62 females $(56.4 \%)$ and 48 males (43.6\%), their age ranged from 7 to 54 years (Figure 1).

Among 110 patients, 47.3\% $(n=52)$ had unilateral Keratoconus while $(\mathrm{n}=58)$ had bilateral Keratoconus. Out of 52 unilateral cases, $24(46.2 \%)$ were females and 28 $(53.8 \%)$ were males. Out of 58 bilateral cases, $38(65.5 \%)$ were females and 20 $(34.5 \%)$ were males.
Munson's sign was present in $68(61.8 \%)$ patients, $42(61.8 \%)$ were females and 26 $(38.2 \%)$ were males, and was not detectable in the remainder of eyes. $61(55.5 \%)$ patients gave a history of eye rubbing out of which 38 $(62.3 \%)$ were females and $23(37.7 \%)$ were males while $49(44.5 \%)$ patients have no such history. Patients showing a family history of keratoconus were $21(19.1 \%)$ out of which 12 $(57.1 \%)$ were females and $9(42.9 \%)$ were males.

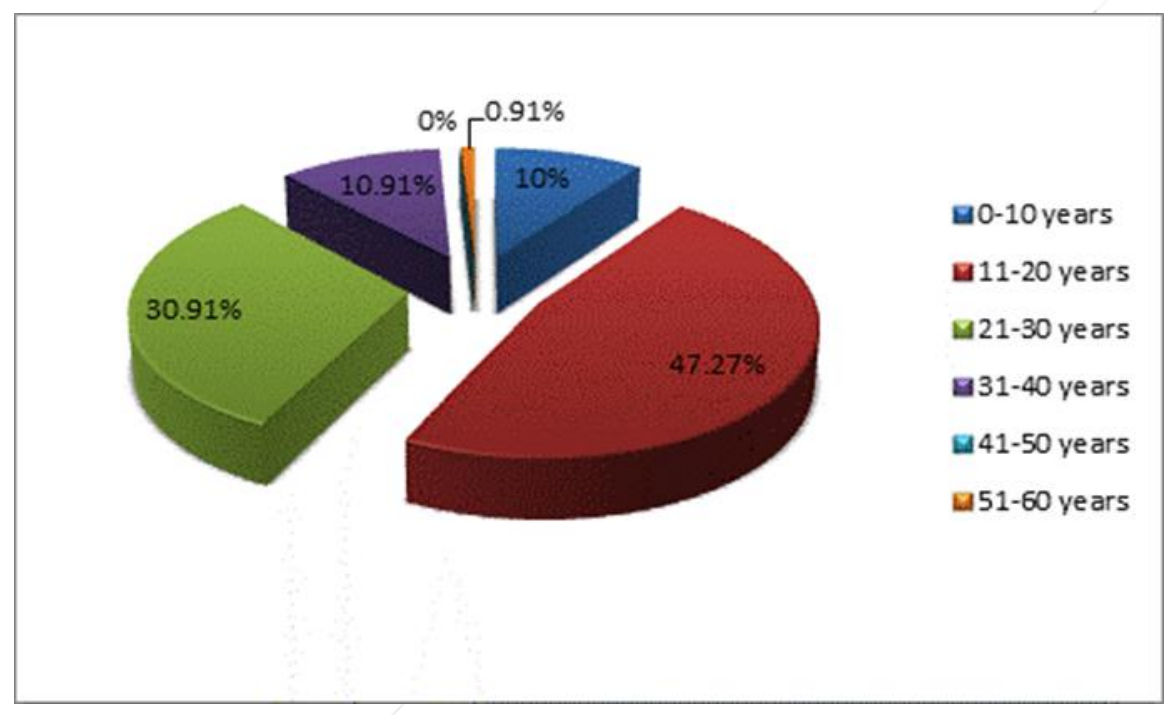

Figure 1. Age distribution of patients of keratoconus

Rizzuti's sign was present in $30(27.3 \%)$ patients out of which $16(53.3 \%)$ were females and $14(46.7 \%)$ were males. Vogt's striae were present in $26(23.6 \%)$ patients out of which $14(53.8 \%)$ were females and 12 $(46.2 \%)$ were males. Fleischer ring was present in $23(20.9 \%)$ patients out of which $12(52.2 \%)$ were females and $11(47.8 \%)$ were males. $29(26.4 \%)$ cases of keratoconus had mild $<45 \mathrm{D}$ k-readings out of which 12 $(41.4 \%)$ were females and $17(58.6 \%)$ were males. $47(42.7 \%)$ cases of keratoconus had moderate $45>52 \mathrm{D}$ k-readings out of which 30 $(63.8 \%)$ were females and $17(36.2 \%)$ were males. $34(30.9 \%)$ cases of keratoconus had severe $>62 \mathrm{D}$ k-readings out of which 20
(58.8\%) were females and $14(41.2 \%)$ were males.

Snellen recordings of eyes of 110 patients were obtained. 11 patients were found to have normal visual acuity (10\%). Moderate vision loss $(6 / 12,6 / 18$, ) was recorded in eyes of 32 patients (29.09\%). High vision loss $(6 / 24,6 / 36,6 / 60$,$) was observed in eyes of 67$ $(60.61 \%)$ patients (Figure 2).

About $19(17.3 \%)$ patients had Atopic syndrome associated with keratoconus out of which $10(52.6 \%)$ were males and $9(47.4 \%)$ were females. $6(5.5 \%)$ patients had Leber's Congenital Amaurosis associated with keratoconus out of which $4(66.7 \%)$ were males and $2(33.3 \%)$ were females. 
Keratoconus is a progressive, noninflammatory disease of the cornea which is characterized by progressive thinning and protrusion of the cornea, which eventually leads to mild or markedly impaired vision due to high myopia and irregular astigmatism [8]. Keratoconus affects typically at puberty and young adults until the third or fourth decade of life with the age between approximately 14 and 35 years, during which the disease can progress or spontaneously arrest. The causes of Keratoconus are multifactorial and are still not completely understood. There are proofs that biochemical, biophysical, and genetic factors play a major contribution in the etiology of this ecstatic corneal disorder. The interplay between environmental and genetic factors is convincing for the development of the disease [9].
In this study, we determined the predominance of Keratoconus in Pakistan. 110 cases of Keratoconus have observed in which the most representative age group was $16-30$, so it is a problem for young people. Keratoconus usually affects both genders in some cases male dominates females [10]. While in others females are more affected [11]. In our study, the disease is more prevalent in females. The disease is initially occurring as unilateral but on progression, it becomes bilateral and asymmetrical. The prevalence of unilateral range from $14.3 \%$ to $41 \%$ when detected by keratometry alone [12] while with computerized topography, unilateral Keratoconus has $0.5 \%$ to $4 \%$ [13]. Bilateral Keratoconus is more common in patients, as in our study the cases of bilateral Keratoconus is high.

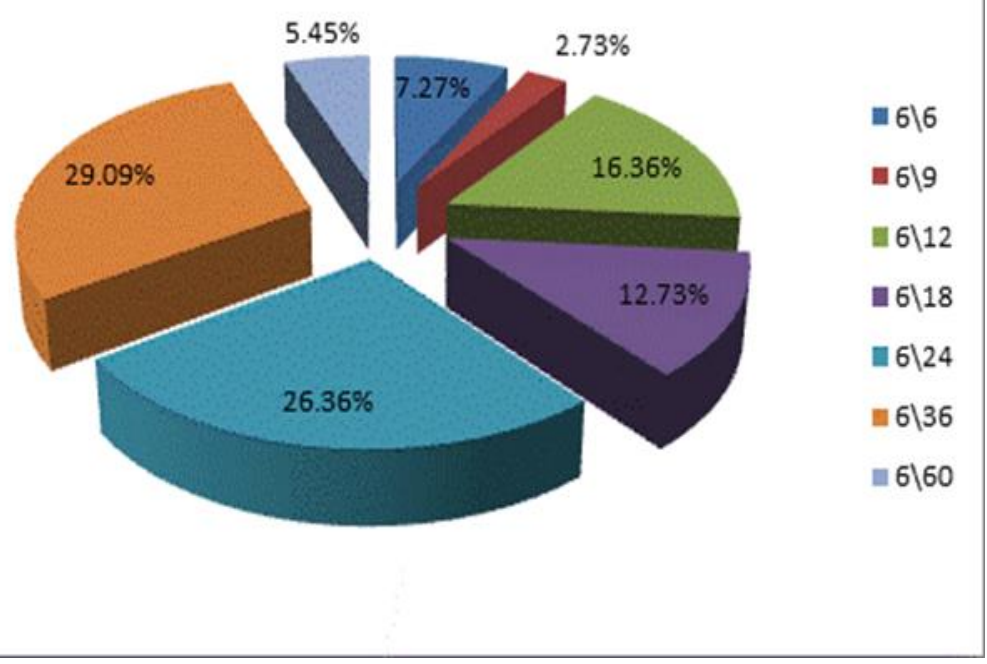

Figure 2. The predominance of visual disability in 110 patients of keratoconus

In our study, 61 patients of Keratoconus gave a positive history of eye rubbing. An association between eye rubbing and Keratoconus has long been described. It is considered a major risk factor. Before the development of Keratoconus, the majority of the patients have a history of habitual eye rubbing. Many reports show that about half of Keratoconus patients rub their eyes [14]. So, it is clear that there is a strong link between the eye rubbing and development of Keratoconus, and has certain differences linked to the eye rubbing is gentle or vigorous. Variation in usual length of rubbing is also notable e.g. Keratoconus patients have more duration of eye rubbing ranging from 
10 -seconds to 180 -seconds than the characteristically less than the 15-seconds duration of rubbing in allergic or infective ocular disorders, in addition, less than 5seconds among people without any eye condition[15].

In this study, $19.1 \%$ of patients showed a family history of Keratoconus while the other studies established that furthermost percentages of general family history were usually lower than $20 \%$. Family history for Keratoconus is due to from either environmental or genetic agents. However, it is not always obvious which of the two is most significant in the pathogenesis of the disease until drawing family pedigree [16]. In our study, Munson's sign was present in 68 patients and also Rizutti's sign was present in 30 patients which occur disease progresses. A Munson's sign, a V-shaped deformation of the lower lid, as well as a bright reflection of the nasal area of the limbus called Rizzuti's sign [17]. We also observed 26 patients who had Vogt's striae and 23 patients had a Fleischer ring. Some of the early biomicroscopic signs were; Fleischer's ring (iron deposition in partial or complete circular form in the epithelium closed to the base of the cornea and Vogt's striae (fine vertical lines produced by compression of Descemet's membrane).

Corneal topography was performed which showed that 29 patients of Keratoconus had mild $<45 \mathrm{D}$ k-readings, 47 patients of Keratoconus had moderate $45>52 \mathrm{D}$ kreadings and 34 patients of Keratoconus had severe $>62 \mathrm{D}$ k-readings. Topography alone cannot lead to a direct diagnosis of Keratoconus, as it must collaborate with clinical signs, symptoms, and thickness optical coherence tomography scans. These diagnostic tools help to differentially distinguish and diagnose Keratoconus from cases of normal irregular astigmatism and corneal dystrophies [18]. Keratoconus may associate with ocular diseases, in our study, we found 19 patients having the atopic syndrome and 6 patients having Leber's Congenital Amaurosis associated with Keratoconus. It has been reported that approximately $35 \%$ of patients with Leber congenital amaurosis, a clinically heterogeneous group of childhood retinal degenerations inherited in an autosomal recessive manner, also suffer from Keratoconus [19].

\section{Conclusion}

Keratoconus is a disease of the cornea in which cornea thins and protrudes, resulting in visual impairment. The disease is much prevalent in Asian population. It usually affects both genders and all ethnic groups. But a higher percentage is observed in females. Both genetic and environmental factors are involved. Keratoconus may associates with ocular disorders like Leber's congenital amaurosis etc and Atopic syndrome as a risk factor.

\section{Authors' contributions}

Conceived and designed the experiments: S Naz, Performed the experiments: N Ibrahim, A Iqbal \& N Sarwar, Analyzed the data: S Sharif, S Sajjad \& A Kaleem, Contributed reagents/ materials/ analysis tools: $\mathrm{S}$ Sharif, Wrote the paper: S Naz \& N Ibrahim.

\section{Acknowledgments}

Hospital administration was cooperative and helps in retrieving complete medical information and history of patients.

\section{References:}

1. Khan SB, Saleem M, Khalid MK, Ahmad I \& Khan MD (2007). Keratoconus and its association with other ocular diseases. PJO 23(4): 212217.

2. Abu-Amero KK, Al-Muammar AM \& Kondkar AA (2014). Genetics of Keratoconus: Where Do We Stand? J Ophthalmol 20(14): 641-708.

3. Shneor E, Millodot M, Blumberg S, Ortenberg I, Behrman S \& GordonShaag A (2013). Characteristics of 244 
patients with keratoconus seen in an optometric contact lens practice. Clin Exp Optomet 96(2): 219-24.

4. Wheeler J, Hauser MA, Afshari NA, Allingham RR \& Liu Y (2012). The genetics of keratoconus: a review. Oxford, England, Microscopy.

5. Choi JA \& Kim MS (2012). Progression of keratoconus by longitudinal assessment with corneal topography. Invest Ophthalmol Vis Sci 53(2): 927935.

6. Vazirani J \& Basu S (2013). Keratoconus: current perspectives. Auckland Clin Ophthalmol 7: 20192030.

7. Wojcik KA, Kaminska A, Blasiak J, Szaflik J \& Szaflik JP (2013). Oxidative stress in the pathogenesis of keratoconus and Fuchs endothelial corneal dystrophy. Inter J Mol Sci 14(9): 19294-19308.

8. Zhang J, Zhang L, Hong J, Wu D \& Xu J (2015). Association of Common Variants in LOX with Keratoconus: A Meta-Analysis. PLoS 10(12): e0145815.

9. IonescuC, Corbu $\mathrm{CG} \&$ Tanase $\mathrm{C}$ (2016). Inflammatory Biomarkers Profile as Micro environmental Expression in Keratoconus. Dis Markers 20(16): 1243819.

10. Lim L, Wei RH, Chan WK \&Tan DTH (2007). Evaluation of keratoconus in Asians: role of Orbscan II and Tomey TMS-2 corneal topography. Am J Ophthalmol 143(3): 390-400.

11. Saini J S, SarohaV, Singh P, Sukhija JS \& Jain AK (2004). Keratoconus in Asian eyes at a tertiary eye care facility. Clin Exp Optomet 87(2): 97-101.
12. Kennedy RH, Bourne WM \& Dyer JA (1986). A 48-year clinical and epidemiologic study of keratoconus. Am J Ophthalmol 101(3): 267-273.

13. Holland DR, Maeda N \& Hannush SB (1997). Unilateral keratoconus: incidence and quantitative topographic analysis. Ophthalmol 104(9): 14091413.

14. Khor WB, Wei RH, Lim L, Chan CM \& Tan DT (2011). Keratoconus in Asians: demographics, clinical characteristics and visual function in a hospital-based population. Clin Exp Ophthalmol 39(4): 299-307.

15. Weed KH, MacEwen C J, Giles T, Low J \& McGhee CNJ (2008). The Dundee University Scottish Keratoconus study: demographics, corneal signs, associated diseases, and eye rubbing. Eye 22(4): 534-541.

16. Gordon-Shaag A, Millodot M, Shneor E \& Liu Y (2015). The Genetic and Environmental Factors for Keratoconus. J Biomed Biotechnol 20(15): 795738.

17. Romero-Jiménez M, SantodomingoRubido J \& Wolffsohn JS (2010). Keratoconus: a review. Contact Lens and Anterior Eye 33(4): 157-166.

18. Munsamy AJ \& Moodley VR (2017). A correlation analysis of cone characteristics and central keratometric readings for the different stages of keratoconus. Indian J Ophthalmol 65(1): 7-11.

19. Elder MJ (1994). Leber congenital amaurosis and its association with keratoconus and keratoglobus. J Pediatr Ophthalmol Strabismus 3(1): 38-40. 